Meta

Journal des traducteurs

Translators' Journal

\title{
L'analyse structurale en traduction poétique
}

\section{Robert Larose}

Volume 23, numéro 1, mars 1978

La traduction poétique

URI : https://id.erudit.org/iderudit/003411ar

DOI : https://doi.org/10.7202/003411ar

Aller au sommaire du numéro

Éditeur(s)

Les Presses de l'Université de Montréal

ISSN

0026-0452 (imprimé)

1492-1421 (numérique)

Découvrir la revue

Citer cet article

Larose, R. (1978). L'analyse structurale en traduction poétique. Meta, 23(1),

47-62. https://doi.org/10.7202/003411ar d'utilisation que vous pouvez consulter en ligne.

https://apropos.erudit.org/fr/usagers/politique-dutilisation/ 


\section{L'analyse structurale en traduction poétique}

Combien de fois nous a-t-on répété que la «forme est intraduisible »?

Au cours des dernières années, dans le cadre d'un séminaire de traductions comparées à l'Université de Montréal, on a tenté de percer le mystère de la «forme » du discours, notamment du discours poétique, en appliquant une méthode structurale mise au point vers 1960 par Roman Jakobson et Claude LéviStrauss. Bien sûr, d'autres chercheurs, par exemple Fónagy, Cohen, Levin et Barthes, ont proposé différents modèles d'analyse structurale, mais jamais dans l'optique de la traduction comparée.

La démarche structurale consiste à relever le plus grand nombre d'éléments du texte de départ à différents niveaux d'analyse (phonologique, morphologique, lexical, syntaxique, sémantique) et d'en dégager une toile d'ensemble. Une telle méthode comme on peut s'en douter, met en veilleuse la réaction du lecteur face au texte, si chère à Riffaterre, de même que la biographie de l'auteur, étrangère au texte, si bien souligné par Rimbaud d'ailleurs, "je est un autre».

Le texte poétique est alors pris comme un tout, indissociable des éléments qui le composent, où chaque niveau contribue à cimenter l'ensemble et ensuite à se dissimuler sous l'《effet poétique ».

Pour illustrer la méthode et ses résultats, nous avons choisi la troisième strophe du poème Israfel (1845) d'Edgar Poe et une traduction de celle-ci par Stéphane Mallarmé. Nous avons choisi cette strophe en raison de la forte concentration d'éléments formels, à première vue intraduisibles. Nous avons cru bon de limiter notre analyse à une seule strophe, car l'analyse du poème en entier dépasserait largement le cadre de cet article.

Troisième strophe, Israfel :

POE

v.16 And they say (the starry choir

v.17 And the other listening things)

v.18 That Israfeli's fire

v.19 Is owing to that lyre

v.20 By which he sits and sings

v.21 The trembling living wire

v. 22 of those unusual strings.

\section{MALLARMÉ}

Ils disent (le cour étoilé et tout ce qui écoute là) que la flamme d'Israfel doit à cette lyre, avec quoi il siège et chante, le frémissement de vie qui se prolonge sur ces cordes extraordinaires. 
Avant de passer aux grilles d'analyse, il faut noter que Mallarmé n'a pas traduit le poème en vers; nous avons adopté une disposition linéaire comparable à la version originale pour faciliter la comparaison.

Les schémas ci-dessous, bien qu'ils n'entendent nullement être exhaustifs, permettent de mieux dégager les éléments de la structure du texte.

\section{GRILLES D'ANALYSE}

\section{A) PROPRIÉTÉS FORMELLES}

\begin{tabular}{lccc}
\hline Niveaux & Aspects & Distribution & Autres \\
\hline Phonographique & $(1)$ & $(2)$ \\
Morphologique (mots-outils) & $(4)$ & $(5)$ & $(3)$ \\
Lexicologique (mots-forts) & $(6)$ & $(7)$ & \\
Syntaxique & La syntaxe (qui comprend la versification) \\
& \multicolumn{3}{c}{ sera analysée séparément } \\
\hline
\end{tabular}

(1) Phonèmes appartenant \pm au système

Graphies \pm conventionnelles

(2) Fréquence des phonèmes

Procédés phoniques (allitération, assonance, etc.)

(3) Eléments considérés comme des régionalismes, dialectalismes, néologismes, clichés, etc., à tous les niveaux d'analyse. Ils ont peu de rendement dans le poème. Aucune de ces remarques n'a été relevée dans la troisième strophe.

(4) Forme des mots-outils : déterminants, pronoms, désinences verbales, adjectifs possessifs et démonstratifs, conjonctions, prépositions, etc. Marques du genre et du nombre pour toutes les catégories grammaticales.

(5) Fréquence des mots-outils.

(6) Forme des mots-forts. Plus précisément nous entendons par mots-forts, les substantifs, verbes, adjectifs qualificatifs (incluant les superlatifs, adjectifs numéraux, verbaux, de comparaison et de couleur) et adverbes.

(7) Fréquence des mots-forts.

Nous empruntons à $\mathrm{P}$. Guiraud, les Caractères statistiques du vocabulaire, p. 20 , les expressions «mots-outils » et «mots-forts »: «On distinguera [...] les mots de structure ou mots-outils (articles, prépositions, pronoms, etc.) et les mots de signification ou mots-forts (substantifs, verbes, adjectifs, adverbes). » 


\section{B) PROPRIETTES SÉMANTIQUES}

\begin{tabular}{lcc}
\hline Niveaux & Dénotations & Connotations \\
\hline Phonographique & $(1)$ & Autres \\
Morphologique (mots-outils) & $(3)$ & $(2)$ \\
Lexicologique (mots-forts) & $(4)$ & La syntaxe (qui comprend la versification) \\
Syntaxique & \multicolumn{2}{c}{ sera analysée séparément }
\end{tabular}

(1) Rare (sauf les cas d'harmonie imitative). Nous empruntons à l'école de Prague la définition de phonème : "Un phonème est le plus petit segment de parole qui, sans avoir de sens en lui-même, est capable de produire une distinction de sens lors de sa commutation avec un autre segment du même ordre », cité par Daniel Delas et Jacques Filliolet, Linguistique et poétique, p. 138.

(2) Références aux interlocuteurs, à d'autres segments du texte, à d'autres textes, à d'autres systèmes sémiologiques, etc., à tous les niveaux d'analyse, incluant les strophes en général.

(3) Mots-outils.

Genre, nombre, etc.

(4) Valeur de base des mots-forts indépendamment des relations syntaxiques.

(5) Aucune observation dans le cas présent.

Les éléments des grilles, choisis arbitrairement, nous permettent d'appliquer une démarche analytique et d'établir une taxinomie des réseaux généralement imperceptibles. Les grilles ne visent qu'à réorganiser le texte et n'entendent nullement présenter plus que ce que le texte contient. Nous nous proposons donc de placer les deux versions côte à côte en vue de rendre leur comparaison plus facile.

\section{POE}

MALLARME

A) Propriétés formelles

1. Niveau phonographique

\subsection{Aspects graphiques}

- $i$ final ajouté à «Israfeli »

- ponctuation : une parenthèse, un tiret et un point final

- absence de $i$ final

- ponctuation : une parenthèse, deux virgules et un point final 


\subsection{Distribution}

Tableau comparatif des paronomases phonologiques :

a) Allitérations ${ }^{2}$ :

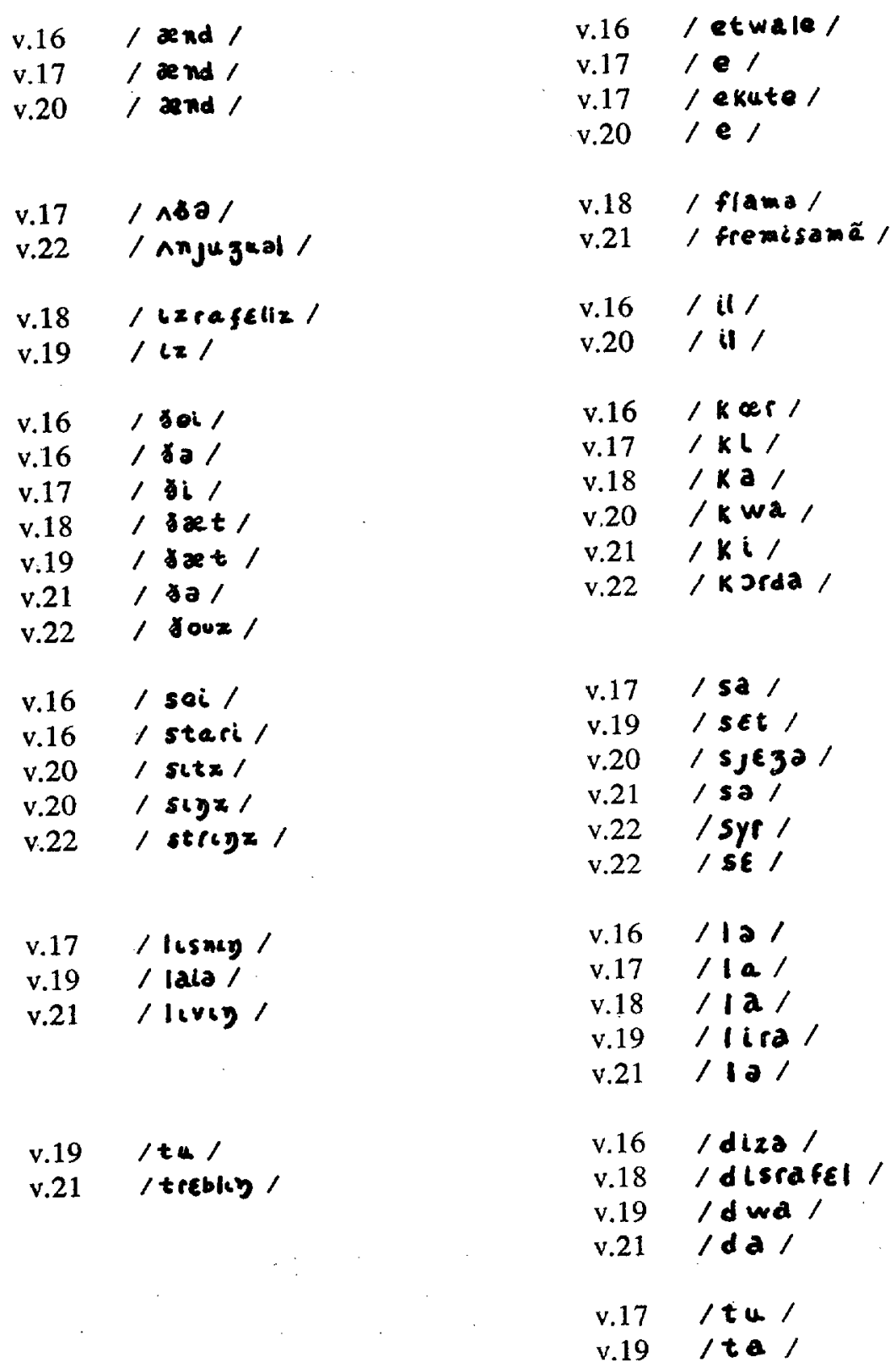

1. Allitération : répétition d'un son ou d'un groupe de sons à l'initiale de plusieurs syllabes ou de plusieurs mots d'un même énoncé. 
b) Consonances ${ }^{2}$ :

v.16 and

v.17, \&nd /

v. 20 / ænd /

$\begin{array}{ll}v .16 & / \mathrm{il} / \\ \mathrm{v} 18 & / \mathrm{d}|s+a f f| / \\ v .20 & / \mathrm{il} /\end{array}$

v.17/ / Isnug /

$\mathrm{v} .16 / k$ er $/$

v.19 / oun /

v.22 / syr /

v.21 / tremblin/

v.21 $/$ lut $/$

v.22 / Ekstrasrdiner/

v.17 / $\theta_{\llcorner\eta z /}$

v.18/lzrafeliz/

v. $20 / s i t z /$

v. 20 /s.

v. $22 /$ ovi /

v.22 / strinz /

v.18/子ชx /

$v .19$ / ชet /

v.16 / $/ \mathrm{ei} /$

v.16 / sei/

$\begin{array}{ll}v .18 & / \text { I / } \\ v .19 & / d w a / \\ v .19 & / t a / \\ v .20 & / k w a /\end{array}$

$v .16 / / a /$

v.17/ekuta /

v.18/Ka/

v.18 / f la ma/

v.19/lira/

v.20 / s.e 3 a $/$

v.20 / Säta/

v.21 / sa/

v.21 / prolszol

v.22 / Karda

$\begin{array}{ll}\text { v.17 } & / \mathrm{ki} / \\ \mathrm{v} .21 & / \mathrm{vi} / \\ \mathrm{v} .21 & / \mathrm{ki} /\end{array}$

v.16 / stari /

v.17 / $/$ il

v. 20 / hi /

2. Consonance : répétition d'un son ou d'un groüpe de sons à la finale de plusieurs syllabes ou de plusieurs mots d'un même énoncé. 
c) Assonances ${ }^{3}$ :

\begin{tabular}{|c|c|c|c|}
\hline \multirow{4}{*}{$\begin{array}{l}\text { v. } 18 \\
\text { v.19 }\end{array}$} & / ræt / & v.18 & /disrafel / \\
\hline & / જæt / & v.19 & $/ s \varepsilon t /$ \\
\hline & & v. 20 & / avek / \\
\hline & & v. 22 & / Ekstrasrdiner/ \\
\hline
\end{tabular}

\begin{tabular}{|c|c|}
\hline v.17 & $/|\operatorname{lsn}| \eta \mid$ \\
\hline v. 17 & $/ \theta \ln x /$ \\
\hline v.19 & / ovin/ \\
\hline v. 20 & I Mits \\
\hline v. 20 & |sttz' \\
\hline v. 20 & $/ \sin z /$ \\
\hline v. 21 & / tremblin \\
\hline v. 21 & $\mid$ ILvon $/$ \\
\hline v. 22 & / strinz \\
\hline
\end{tabular}

2. Niveau morphologique (mots-outils)

2.1. Aspects

- Sept mots se terminent en -ing : Nul

v.17 listening thing(s)

v.19 owing

v.20 $\operatorname{sing}(\mathrm{s})$

v.21 trembling

living

v.22 string(s)

- Narration à la troisième personne

\begin{tabular}{|c|c|c|c|}
\hline Verbes & temps des verbes & verbes & temps des verbes \\
\hline $\begin{array}{l}\text { say } \\
\text { is owing }\end{array}$ & $\begin{array}{l}\text { indicatif présent } \\
\text { présent de la forme } \\
\text { progressive }\end{array}$ & $\begin{array}{l}\text { disent } \\
\text { écoute }\end{array}$ & $\begin{array}{l}\text { indicatif présent } \\
\text { indicatif présent }\end{array}$ \\
\hline $\begin{array}{l}\text { sits } \\
\text { sings }\end{array}$ & $\begin{array}{l}\text { indicatif présent } \\
\text { indicatif présent }\end{array}$ & $\begin{array}{l}\text { doit } \\
\text { siège } \\
\text { chante } \\
\text { prolonge }\end{array}$ & $\begin{array}{l}\text { indicatif présent } \\
\text { indicatif présent } \\
\text { indicatif présent } \\
\text { indicatif présent }\end{array}$ \\
\hline
\end{tabular}

\subsection{Distribution}

$\begin{array}{lll}3 & \text { - conjonction - } & 2 \\ 3 & \text { - préposition - } & 3 \\ 3 & \text { - article défini - } & 3\end{array}$
Assonance : répétition de la voyelle à la finale d'un mot ou d'un groupe rythmique
suivie d'une consonne. 


$\begin{array}{lll}2 & \text { - pronom personnel - } & 2 \\ 2 & \text { - pronom relatif - } & 4 \\ 0 & \text { - pronom indéfini - pronom démonstratif - } & 1 \\ 0 & \text { - pronom réfléchi - } & 1 \\ 0 & \text { - article partitif - } & 1 \\ 0 & \text { - adjectif démonstratif - } & 2 \\ 2 & \end{array}$

3. Niveau lexicologique (mots-forts)

\subsection{Aspects}

Tous les adjectifs se terminent en -ing Nul

\subsection{Distribution}

$\begin{array}{lll}4 & \text { - verbum finitum - } & 6 \\ 1 & \text { - verbe auxiliaire - } & 0 \\ 0 & \text { - adverbe - } & 1 \\ 6 & \text { - nom commun - } & 6 \\ 1 & \text { - nom propre - adjectif qualificatif - } & 1 \\ 3 & \text { - adjectif verbal - } & 1 \\ 3 & \text { - participe passé adjectif - } & 0 \\ 0 & \end{array}$

B) Propriétés sémantiques

1. Niveau phonographique

\subsection{Dénotations}

- Mabbott ${ }^{4}$ explique l'addition du $i$ dans \& Israfeli » de la façon suivante : \&Israfeli means grammatically «my Israfel » but Dr. John L. Mish tells me the usage is almost unknown, and Poe's note in which he thought «Israfeli » a variant nominative makes one feel that here Poe builded (sic) better than he knew ».

- Les propriétés acoustiques de -ing correspondent à l'aspect musical de la strophe.

\begin{abstract}
Mallarmé a traduit Israfeli par
\end{abstract} «Israfel 》

4. Thomas Olive Mabbott, Collected Works of Edgar Allan Poe, Cambridge (Mass.), Harvard University Press, 1969, p. 178. 


\subsection{Connotations}

- Parallélisme son/sens :

allitérations: + auditif

$$
\begin{aligned}
& \text { sings } \\
& \text { strings } \\
& \text { listening } \\
& \text { lyre }
\end{aligned}
$$

consonances /assonances : \pm auditif

$$
\begin{aligned}
& \text { sing(s) } \\
& \text { string(s) } \\
& \text { listening } \\
& \text { choir } \\
& \text { lyre } \\
& \text { wire }
\end{aligned}
$$

- Effet archaïsant du $i$ terminal dans

«Israfeli "

2. Niveau morphologique (mots-outils)

\subsection{Dénotations}

$\mathrm{Nul}$

Nul

\subsection{Connotations}

$\mathrm{Nul}$

Nul

3. Niveau lexicologique (mots-forts)

\subsection{Dénotations}

$\mathrm{Nul}$

- Mallarmé a commis un faux sens en traduisant «choir» par «cœur». Il s'agit peut-être d'une coquille.

\subsection{Connotations}

- Paradigme + musical : choir lyre

sings

wire

strings

- Métonymie : «strings 》 (à remarquer le bathos de «unusual strings » qui fait suite à une série d'adjectifs très recherchés).

- Métaphores (personnification) :

$\begin{array}{ll}\begin{array}{l}\text { animé } \\ \text { choir }\end{array} & \begin{array}{l}\text { inanimé } \\ \text { listening }\end{array} \\ \end{array}$

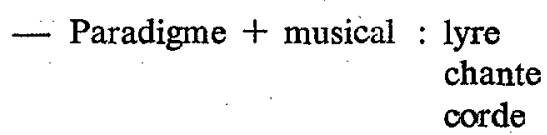

- Mallarmé rattache «frémissement 》 à «flamme », alors qu'en anglais «trembling». se rapporte à «lyre» et non à \& fire ».

$\begin{array}{ll}\text { animé } & \text { inanimé } \\ \text { Israfel } & \text { flamme } \\ \text { frémissement } & \end{array}$




$\begin{array}{llll}\text { say } & \text { things } & \text { de vie } & \text { corde } \\ \text { trembling } & & & \\ \text { living } & \text { wire } & \\ \text { Israfeli } & \text { fire } & \end{array}$

C) SYNTAXE ET VERSIFICATION
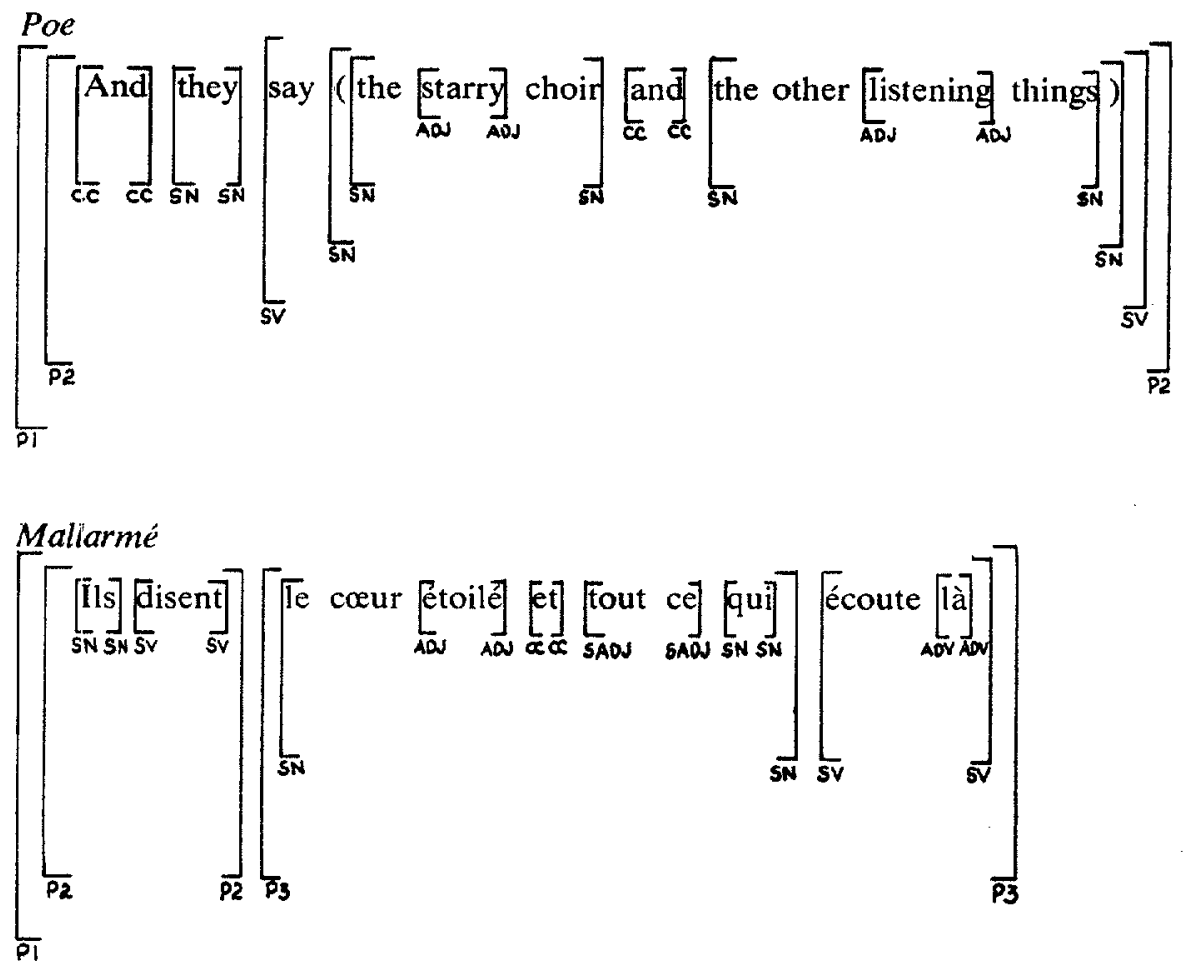

Poe

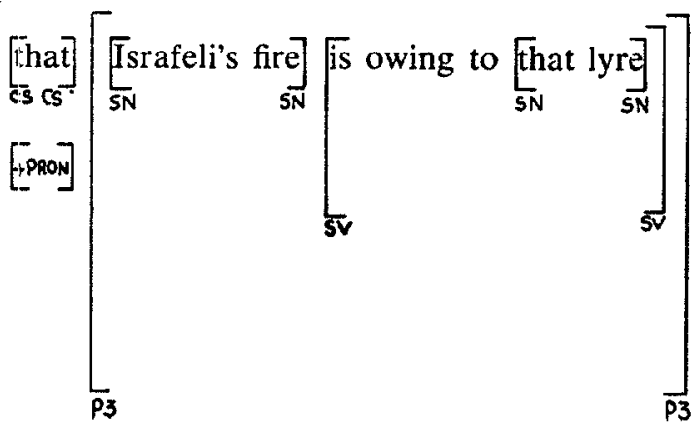



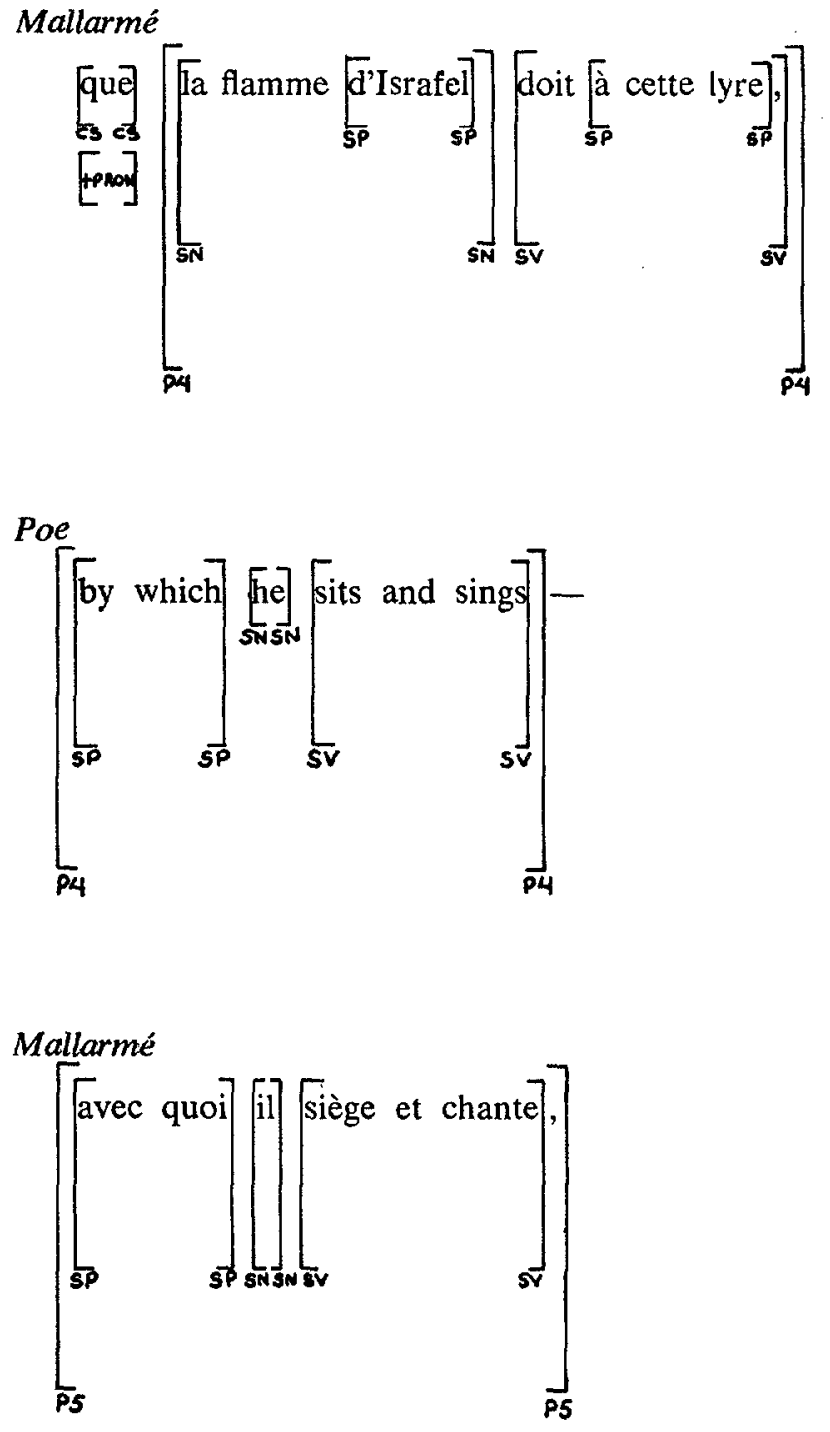

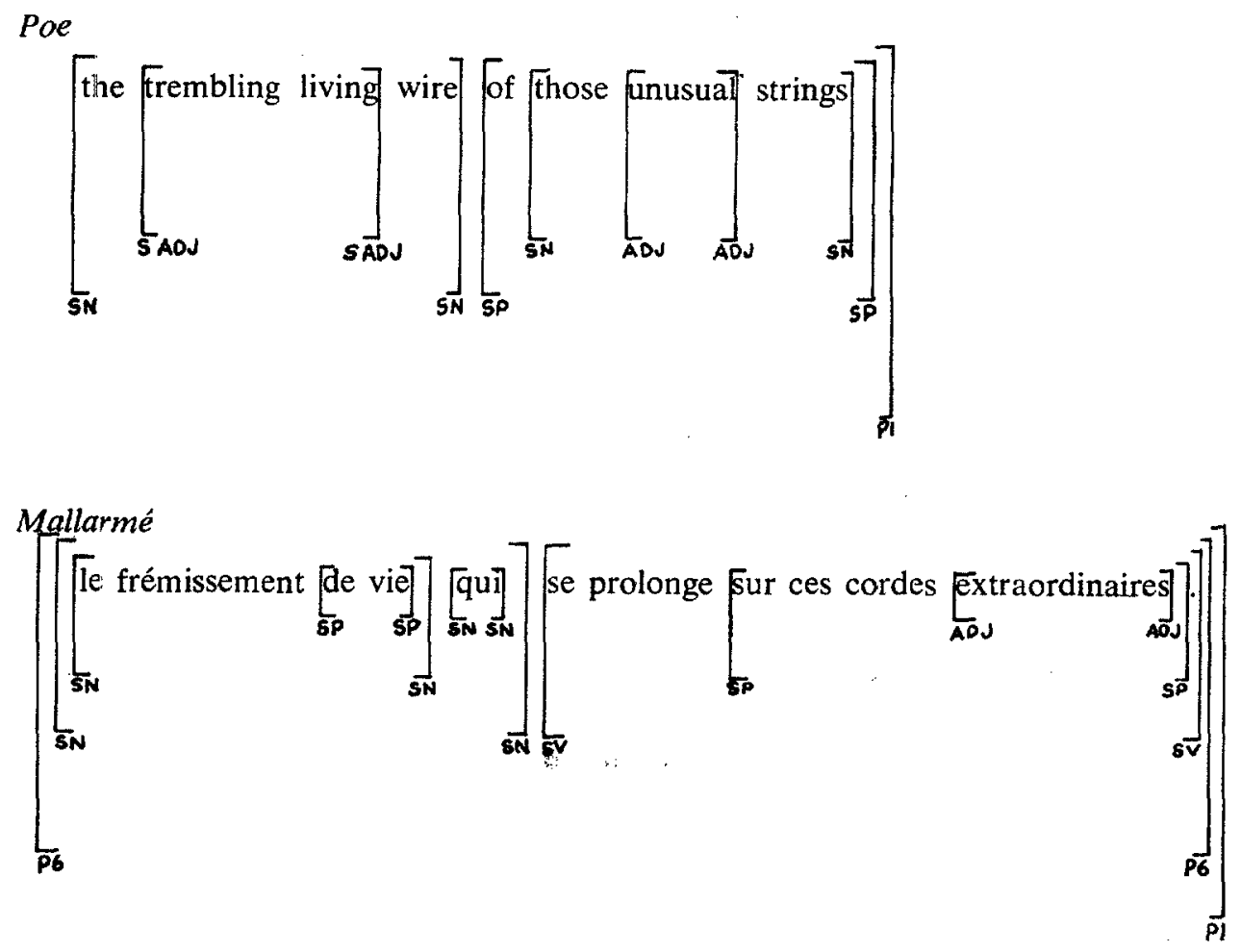

\section{Syntaxe}

POE : La strophe III $\left(\mathrm{P}_{1}\right)$ commence par une conjonction de coordination (And) et englobe trois phrases qui se succèdent en structure profonde. Le segment incomplet ( the trembling living wire of those unusual strings $»$ ), en apposition à la fin de $\mathrm{P}_{1}$, comprend un syntagme adjectival inhabituel (" trembling living ») où les propriétés grammaticales (les deux mots déterminent « wire ») renforcent le croisement phonico-sémantique (lien consonantique en ing et rapport animé/ inanimé entre les déterminants et le nom «wire $\gg) . P_{2}$ possède un $S N$ en apposition (« the starry choir and the other listening things »).

MALlaRmé : La traduction de Mallarmé comprend une phrase en structure de surface $\left(P_{1}\right)$ et 5 en structure profonde $\left(P_{2} \grave{a} P_{6}\right)$. $P_{6}$, cependant, nous semble d'une construction douteuse et, comme nous l'avons souligné plus haut, établit un rapport avec \& flamme», tandis que le SN en apposition dans la version anglaise explique \& lyre $»$.

\section{Versification}

Comme Mallarmé n'a pas traduit le poème en vers, la comparaison sur le plan de la versification s'avère impossible. Néanmoins, le septain se présente de la façon suivante. 


$\begin{array}{lllll}\text { v.16 } & \text { trimètre } & \text { anapeste } & \text { iambe } & \text { iambe } \\ \text { v.17 } & \text { trimètre } & \text { anapeste } & \text { iambe } & \text { iambe } \\ \text { v.18 } & \text { trimètre } & \text { iambe } & \text { iambe } & \text { iambe } \\ \text { v.19 } & \text { trimètre } & \text { iambe } & \text { iambe } & \text { iambe } \\ \mathrm{v} .20 & \text { trimètre } & \text { iambe } & \text { iambe } & \text { iambe } \\ \mathrm{v} .21 & \text { trimètre } & \text { iambe } & \text { iambe } & \text { iambe } \\ \mathrm{v} .22 & \text { trimètre } & \text { iambe } & \text { iambe } & \text { iambe }\end{array}$

\begin{tabular}{lcl|} 
Rimes & externes & : \\
/aij/ & v.16 & choir \\
& v.18 & fire \\
& v.19 & lyre \\
& v.21 & wire
\end{tabular}

rimes vocaliques

rimes riches

\section{TRADUCTION STRUCTURALE}

Il ressort de l'analyse que la traduction de Mallarmé comporte certaines lacunes sur le plan structural. Sans vouloir porter un jugement de valeur (Dieu nous en préserve !) sur la traduction de Mallarmé, nous avons l'intention à titre expérimental de proposer dans la mesure du possible une traduction qui respecterait davantage la texture formelle du poème de départ dont Mallarmé n'a pas tenu compte.

Traduction structurale proposée

v.16 And they say (the starry choir) / Et l'on raconte (les astres en chœur

v.17 And the other listening things / Qui écoutent comme toutes les autres choses)

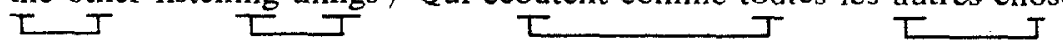

v.18 That Israfeli's fire / Qu'Israfeli et sa lueur

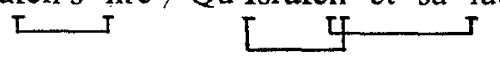

v.19 Is owing to that lyre / Puisent leur éclat dans cette lyre

v.20 By which he sits and sings/Qu'il pince tout en chantant

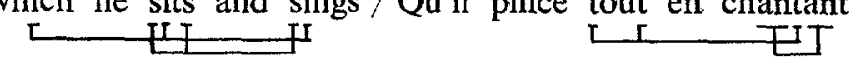

v.21 The trembling living wire / les accents vivants qui frémissent, qui vibrent

v.22 Of those unusual strings / de ces irréelles chanterelles 


\section{Commentaires}

Nous avons, dans la mesure du possible, transposé, dans notre traduction, les réseaux phoniques de la strophe de l'original. Les rimes externes ne suivent pas le modèle abaabab de l'original. Mais, nous avons tenté de combler cette lacune en établissant des convergences d'un autre ordre. Par exemple, les vers 16 (rime visuelle), 17,20 et 22 sont liés entre eux par le $/ \int /$, ou ch, tandis que les vers 18 et 19 sont reliés par le / $1 /$. Le vers 21 , en retour, a été modifié pour tenir compte des deux adjectifs verbaux anglais ( trembling living»), lacune acceptable d'ailleurs si l'on considère le caractère irrégulier de la versification dans l'ensemble du poème. Au vers 16 , «les astres en chœur » conservent le rapport animé/inanimé de la version anglaise. Au vers suivant, Poe maintient ce rapport ( ( listening things »). Il est préférable, selon nous, de rendre «things » par «choses » plutôt que par «tout 》(Mallarmé), trop vague ici. Au vers 18, nous avons conservé la graphie archaïque de «Israfeli» et, bien que «lueur» ne soit pas une traduction exacte de «fire », "éclat» au vers 19 nous permet de garder toute l'intensité du mot anglais. La conjonction de coordination « et» établit un autre rapport animé/inanimé, procédé de personnification amplement utilisé par Poe dans le poème. Traduire littéralement «sits and sings» (v.20) nous semblait quelque peu maladroit. Nous avons donc transposé au niveau sémantique l'allitération en substituant "pince» à "sits», plus dynamique en l'occurrence. Aussi, au vers suivant, «trembling living» a été l'objet d'une double transposition : d'abord au niveau syntaxique («qui frémissent, qui vibrent »), puis au niveau lexical ( trembling/frémissent » et $\&$ living/vivants, vibrent »). Dans ce dernier cas, le lien consonantique de "trembling living » est remplacé par l'allitération « vivants/vibrent» et, sur le plan morphologique, par la terminaison des verbes ("frémissent/vibrent»). Il est à noter que le mot " accents », comme «wire», appartient au paradigme musical. Le bathos du dernier vers ( unusual strings ») a été remplacé par une rime interne («irréelles chanterelles 》) qui s'intègre elle aussi dans le paradigme musical de la strophe. $\mathrm{La}$ «chanterelle» désigne la corde la plus fine, ayant le son le plus aigu, dans un instrument à cordes. Le mot constitue un écho savant qui nous renvoie au mot archaïsant «Israfeli » (v.18).

D'après les résultats de notre analyse, certains éléments de la forme sont apparemment transposables d'une langue à une autre. Les limites en sont bien sûr les règles de fonctionnement et le lexique propres à chaque langue. Que l'on dise, par exemple, qu'un phonème ne puisse avoir la même valeur d'une langue à l'autre, c'est méconnaître la science linguistique et confondre l'arbitraire et la motivation des signes. Bien qu'il $\mathrm{y}$ ait différence possible entre les règles de réalisations phonologiques $\mathrm{du} / \mathrm{r} /$ français et $\mathrm{du} / \mathrm{r} /$ anglais, leur transposition, jusqu'à un certain point, reste faisable.

Il faut admettre, cependant, que la traduction totale est impossible en raison de l'immanence des codes d'une langue, irréductibles les uns des autres. Les résultats de notre analyse offrent pourtant le double avantage de servir de 
paramètres en traduction comparée et d'outils en vue de traduire le plus fidèlement possible un texte, à tous les niveaux d'analyse.

Robert Larose et Daniel Slote

ISRAFEL

And the angel Israfel,

whose heart-strings are a lute, and who has the sweetest voice of all God's creatures. - KORAN.

In Heaven a spirit doth dwell

"Whose heart-strings are a lute;

None sing so wildly well

As the angel Israfel,

And the giddy stars (so legends tell)

Ceasing their hymns, attend the spell

Of his voice, all mute.

Tottering above

In her highest noon,

The enamoured moon

Blushes with love,

While, to listen, the red levin

(With the rapid Pleiads, even,

Which were seven,)

Pauses in Heaven.

And they say (the starry choir And the other listening things)

That Israfeli's fire

Is owing to that lyre By which he sits and sings -

The trembling living wire

Of those unusual strings.

But the skies that angel trod,

Where deep thoughts are a duty -

Where Love's a grown-up God -

Where the Houri glances are

Imbued with all the beauty

Which we worship in a star.

Therefore, thou art not wrong,

Israfeli, who despisest

An unimpassioned song;

To thee the laurels belong,

Best bard, because the wisest !

Merrily live, and long! 
The ecstasies above

With thy burning measures suit -

Thy grief, thy joy, thy hate, thy love,

With the fervour of thy lute -

Well may the stars be mute!

Yes, Heaven is thine; but this

Is a world of sweets and sours;

Our flowers are merely - flowers,

And the shadow of thy perfect bliss

Is the sunshine of ours.

If I could dwell

Where Israfel

Hath dwelt, and he where I,

He might not sing so wildly well

A mortal melody,

While a bolder note than this might swell

From my lyre within the sky.

\section{ISRAFEL}

Dans le ciel habite un esprit «dont les fibres du cœur font un luth ». Nul ne chante si étrangement bien - que l'ange Israfel, et les étoiles irrésolues (au dire des légendes) cessant leurs hymnes, se prennent au charme de sa voix, muettes toutes.

Vacillante et lointaine à sa plus haute heure, la lune enamourée rougit de passion; alors, pour écouter, la vermeille clarté ainsi que les rapides Pléiades, elles-mêmes, toutes les sept, fait une pause dans les Cieux.

Ils disent (le cœur étoilé et tout ce qui écoute là) que la flamme d'Israfel doit à cette lyre, avec quoi il siège et chante, le frémissement de vie qui se prolonge sur ces cordes extraordinaires.

Mais cet ange a foulé le firmament, où de profondes pensées sont un devoir - où l'Amour est un dieu dans sa force - où les cillades des houris possèdent toute la beauté que l'on adore dans une étoile.

Voilà pourquoi tu n'as pas tort, Israfel, que ne satisfait pas un chant impossible ; à toi appartiennent les lauriers, ô Barde le meilleur, étant le plus sage! Vis joyeusement et longtemps ! et longtemps!

Les célestes extases d'en haut, certes, vont bien à tes brûlantes mesures; ta peine, ta joie, ta haine, ton amour, à la ferveur de ton luth - les étoiles peuvent être muettes.

Oui, le ciel est à toi, mais chez nous est un monde de douceurs et d'amertumes; nos fleurs sont simplement - des fleurs; et l'ombre de ta félicité parfaite est le sommeil de la nôtre.

Si je pouvais habiter où Israfel habite et que lui me fût, il se pourrait qu'il ne chantât pas si étrangement bien une mélodie mortelle; tandis qu'une note plus forte que celle-ci peut-être roulerait de ma lyre dans le Ciel.

Nous désirons remercier MM. Nathan Ménard, Laurent Santerre et Rajendra Singh, professeurs au Département de linguistique et philologie de l'Université de Montréal, pour leur précieuse collaboration. 


\section{BIBLIOGRAPHIE}

ALLEN, Harvey, Israfel : The Life and Times of Edgar Allen Poe, New York, Rinehart. BLINKENBERG, Andreas, lordre des mots en français moderne, Copenhague, Bianco Lunos Bogtrykkeri, 1928.

COHEN, Jean, Structure du langage poétique, Paris, Flammarion, 1971.

"La comparaison poétique ", Langages, 12, 43-51.

«Théorie de la figure », Communication, 16, 1970, 3-25.

CORVEZ, Maurice, les Structuralistes, Paris, Aubier-Montaigne, 1969.

DAVIDSON, Gustav, « Poe's Israfel », Literary Review, Fairleigh Dickinson University, 12 , 1968, 86-91.

DUBOIS, Jean, Mathée GIACOMO, Louis GUESPIN, Christiane MARCELLESI, JeanBaptiste MARCELLESI et Jean-Pierre MEVEL, Dictionnaire de linguistique, Paris, Larousse, 1973.

DUCROT, Oswald, Qu'est-ce que le structuralisme? I. Le structuralisme en linguistique, Paris, Seuil, 1973.

FONAGY, I., "Le langage poétique : forme et fonction ", Diogène, 51, 1965, 72-113.

FRAISSE, P., les Structures rythmiques, Paris, Ed. Erasme, 1956.

GREIMAS, A.J., Essais de sémiologie poétique, Paris, Larousse, 1972.

HALLIBURTON, David, Edgar Allan Poe : A Phenomenological View, Princeton University Press, 1973.

JAKOBSON, Roman, Essais de linguistique générale, Paris, Seuil, 1970.

Questions de poétique, Paris, Seuil, 1973.

MABBOTT, Thomas O., « Poe's Israfel », Explicator, II June 1944, 57.

MALLARMÉ, Stéphane, Euvres complètes, Pléiade, Gallimard, 1961.

MOUNIN, Georges, les Problèmes théoriques de la traduction, Paris, Gallimard, 1971.

RIFFATERRE, Michel, Essais de stylistique structurale, Paris, Flammarion, 1971.

RUWET, Nicolas, Théorie syntaxique et syntaxe du français, Paris, Seuil, 1972.

Langage, musique, poésie, Paris, Seuil, 1972.

SUBERVILLE, Jean, Histoire et théorie de la versification française, Paris, Les Éd. de l'Ecole, s.d.

TODOROV, Tzvetan, Qu'est-ce que le structuralisme? 2. Poétique, Paris, Seuil, 1973.

WERNER, W.L., "Poe's Israfel ", Explicator, II April 1944, 44. 